\title{
Recovering entanglement by local operations
}

\author{
A. D’Arrigo a,b,c,*, R. Lo Franco d,c, G. Benenti ${ }^{\mathrm{e}, \mathrm{f}}$, \\ E. Paladino ${ }^{\mathrm{b}, \mathrm{a}, \mathrm{c}, \mathrm{g}}, \mathrm{G}$. Falci ${ }^{\mathrm{b}, \mathrm{a}, \mathrm{c}, \mathrm{g}}$ \\ a CNR-IMM UOS Università (MATIS), Consiglio Nazionale delle Ricerche, Via Santa Sofia 64, \\ 95123 Catania, Italy \\ ${ }^{\mathrm{b}}$ Dipartimento di Fisica e Astronomia, Università degli Studi Catania, Via Santa Sofia 64, \\ 95123 Catania, Italy \\ ${ }^{\mathrm{c}}$ Centro Siciliano di Fisica Nucleare e Struttura della Materia (CSFNSM), Via Santa Sofia 64, \\ 95123 Catania, Italy \\ d Dipartimento di Fisica e Chimica, Università di Palermo, via Archirafi 36, 90123 Palermo, Italy \\ e CNISM and Center for Nonlinear and Complex Systems, Università degli Studi dell'Insubria, Via Valleggio \\ 11, 22100 Como, Italy \\ ${ }_{\mathrm{f}}^{\mathrm{f}}$ Istituto Nazionale di Fisica Nucleare, Sezione di Milano, via Celoria 16, 20133 Milano, Italy \\ ${ }^{\mathrm{g}}$ Istituto Nazionale di Fisica Nucleare, Sezione di Catania, Viale S. Sofia 64, 95123 Catania, Italy
}

\section{A R T I C L E I N F O}

Article history:

Received 1 February 2014

Accepted 18 July 2014

Available online 23 July 2014

\section{Keywords:}

Quantum information

Entanglement

Decoherence

Open quantum systems

\begin{abstract}
A B S T R A C T
We investigate the phenomenon of bipartite entanglement revivals under purely local operations in systems subject to local and independent classical noise sources. We explain this apparent paradox in the physical ensemble description of the system state by introducing the concept of "hidden" entanglement, which indicates the amount of entanglement that cannot be exploited due to the lack of classical information on the system. For this reason this part of entanglement can be recovered without the action of non-local operations or back-transfer process. For two noninteracting qubits under a low-frequency stochastic noise, we show that entanglement can be recovered by local pulses only. We also discuss how hidden entanglement may provide new insights about entanglement revivals in non-Markovian dynamics.
\end{abstract}

(c) 2014 Elsevier Inc. All rights reserved.

\footnotetext{
* Corresponding author at: CNR-IMM UOS Università (MATIS), Consiglio Nazionale delle Ricerche, Via Santa Sofia 64, 95123 Catania, Italy.

E-mail addresses: antonio.darrigo@dmfci.unict.it, ant.darrigo@gmail.com (A. D’Arrigo).
} 


\section{Introduction}

Entanglement, arguably the most peculiar feature of quantum mechanics, plays a key role in several quantum information and communication applications, including teleportation, quantum dense coding, private key distribution, and reduction of communication complexity [1-4]. To work properly, all the above tasks generally require pure maximally entangled states. Since entanglement cannot be generated by Local Operations and Classical Communication (LOCC), entangled states must be generated somewhere, and then they have to be distributed among different parties, possibly far away from each other (transmission) [3,4]. Once entanglement has been distributed, it can be used immediately or stored for later use (storage). Systems physically supporting entangled states, unavoidably interact with the environment, both during transmission and storage, and therefore undergo noisy processes that deteriorate entanglement. Quantification of entanglement losses is thereby necessary for all practical purposes.

For a pure state $\rho=|\psi\rangle\langle\psi|$, bipartite entanglement between subsystems $A$ and $B$ is unambiguously defined as the entropy of entanglement $E(|\psi\rangle\langle\psi|)=S\left(\rho_{A}\right)=S\left(\rho_{B}\right)$, where $S\left(\rho_{i}\right)$ is the von Neumann entropy of one of the two reduced states, $\rho_{A}=\operatorname{Tr}_{B} \rho$ and $\rho_{B}=\operatorname{Tr}_{A} \rho$. The quantification of entanglement for mixed states is a much more complicated and still open problem [3,4]. The difficulty roots in the fact that a mixed state $\rho$ may be decomposed into an ensemble of pure states $\rho=\sum_{i} p_{i}\left|\psi_{i}\right\rangle\left\langle\psi_{i}\right|$, with $p_{i}>0$ and $\sum_{i} p_{i}=1$, in infinite different ways. The arbitrariness of the decomposition renders any quantification of mixed-state entanglement cumbersome, since it requires an optimization over all possible decompositions.

In this article, we address the issue of the occurrence of entanglement revivals of a bipartite system, initially prepared in an entangled state, when the two subsystems are noninteracting and affected by local independent classical noise sources and local operations (see Fig. 1(a)). In the absence of non-local operations, entanglement cannot be generated neither back-transferred to the system from the classical environment. Nevertheless, during the system dynamics, entanglement quantified by some measure $E$ may start to increase at some time $\bar{t}[5,6]$ as illustrated in Fig. 1(b). As we will explain, the increase of entanglement must be attributed to the manifestation of pre-existing quantum correlations, that were already present before $\bar{t}$. The density operator formalism does not capture the presence of these quantum correlations, thus they are in some sense hidden. Here we point out that the existence of these correlations is enlightened if the system is described as a physical ensemble of states and we introduce the concept of hidden entanglement.

This paper is structured as follows. In Section 2 we introduce a definition of hidden entanglement (HE) and illustrate the usefulness of this concept by a simple example. In Section 3 we show that HE between two noninteracting qubits subject to a non-Markovian stochastic process can be recovered by local pulses (acting only on one qubit). The nature of the observed entanglement revivals and the relation of this phenomenon with the environment being classical or quantum, are clarified. In Section 4 we critically discuss some key points related to the definition of HE. In particular, we show that entanglement recovery does not violate the monotonicity axiom: entanglement cannot increase under LOCC $[3,4,7]$. We draw our conclusions in Section 5.

\section{Hidden entanglement}

Let us consider a bipartite system described by an ensemble of states $\mathcal{A}=\left\{\left(p_{i},\left|\psi_{i}\right\rangle\right)\right\}$. That is, we know the statistical distribution of the bipartite pure states $\left\{\left|\psi_{i}\right\rangle\right\}$, occurring with probabilities $\left\{p_{i}\right\}$, so that $\rho=\sum_{i} p_{i}\left|\psi_{i}\right\rangle\left\langle\psi_{i}\right|$, but the state of any individual system in the ensemble is unknown. The average entanglement of $\mathcal{A}$ is defined as [7-10]:

$$
E_{\mathrm{av}}(\mathcal{A})=\sum_{i} p_{i} E\left(\left|\psi_{i}\right\rangle\left\langle\psi_{i}\right|\right)
$$

If each system in the ensemble evolves during time $t$ under LOCC, the maximum amount of entanglement of the corresponding density operator $\rho(t)$ can never overcome the initial value $E_{\mathrm{av}}(\mathcal{A})$. This statement can be proved by the following simple argument. Suppose Charlie prepares a bipartite 

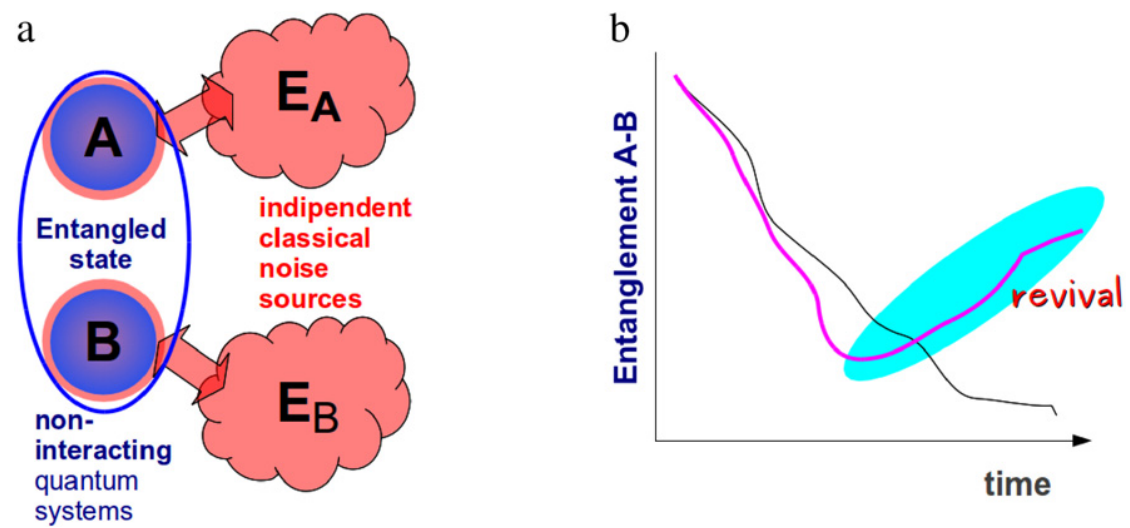

Fig. 1. (a) Two quantum systems, $A$ and $B$, initially prepared in an entangled state are transferred to different locations where they do not interact each other and are subject to local independent classical noise sources and local operations. (b) The thin (black) line describes the most usual entanglement behavior of the considered system. In this article we will point out the possibility that this system may exhibit a non monotonic entanglement behavior without the action of any non-local control, qualitatively sketched by the thick (magenta) line. (For interpretation of the references to color in this figure legend, the reader is referred to the web version of this article.)

system in a (possibly entangled) pure state of the ensemble $\mathcal{A}$. Then he sends one half of the system to Alice and the other half to Bob through noiseless quantum channels. Alice and Bob communicate only by a noiseless classical channel. Charlie repeats this operation $N$ times. Among these, a certain number $M_{j}$ of times Alice and Bob deal with the state $\left|\psi_{j}\right\rangle$. If Alice (or Bob) receives from Charlie the classical information about which state he sent each time, in the limit of large $N$ Alice and Bob can distil - by only using LOCC - up to $M_{j} E\left(\left|\psi_{j}\right\rangle\left\langle\psi_{j}\right|\right)$ maximally entangled states from the $M_{j}$ states $\left|\psi_{j}\right\rangle$ at their disposal [7]. Distillable entanglement is in fact the entropy of entanglement for pure bipartite states. Therefore, the maximum entanglement that Alice and Bob can distil per pair, by using classical information from Charlie, is

$$
\lim _{N \rightarrow \infty} \frac{1}{N} \sum_{i} M_{i} E\left(\left|\psi_{i}\right\rangle\left\langle\psi_{i}\right|\right)=\sum_{i} p_{i} E\left(\left|\psi_{i}\right\rangle\left\langle\psi_{i}\right|\right),
$$

which is just the average entanglement of Eq. (1) $\left(\lim _{N \rightarrow \infty} \frac{M_{i}}{N} \equiv p_{i}\right)$.

We define the hidden entanglement (HE) of the ensemble $\mathcal{A}=\left\{\left(p_{i},\left|\psi_{i}\right\rangle\right)\right\}$ as the difference between the average entanglement of the ensemble and the entanglement [3,4] of the state $\rho=\sum_{i} p_{i}\left|\psi_{i}\right\rangle\left\langle\psi_{i}\right|$ quantified by any convex measurement $E(\rho)$ (reducing to the entropy of entanglement for pure states), that is ${ }^{1}$

$$
\begin{aligned}
E_{h}(\mathcal{A}) & \equiv E_{\mathrm{av}}(\mathcal{A})-E(\rho) \\
& =\sum_{i} p_{i} E\left(\left|\psi_{i}\right\rangle\left\langle\psi_{i}\right|\right)-E\left(\sum_{i} p_{i}\left|\psi_{i}\right\rangle\left\langle\psi_{i}\right|\right) .
\end{aligned}
$$

Due to convexity, $E_{h}$ is always larger than or equal to zero. The meaning of HE Eq. (3) is clear: It is the entanglement that cannot be exploited as a resource due to the lack of knowledge about which state of the mixture we are dealing with (see Fig. 2). Such entanglement can be recovered (unlocked $[8,11,12])$ once this classical information is provided, without the help of any non local operation. We remark, as it is clear from the definition Eq. (3), that HE is associated to the specific quantum ensemble description of the system state. We will refer to situations where the system dynamics

1 Note that in Ref. [8] the expression "hidden entanglement" is used with a different meaning. 
a
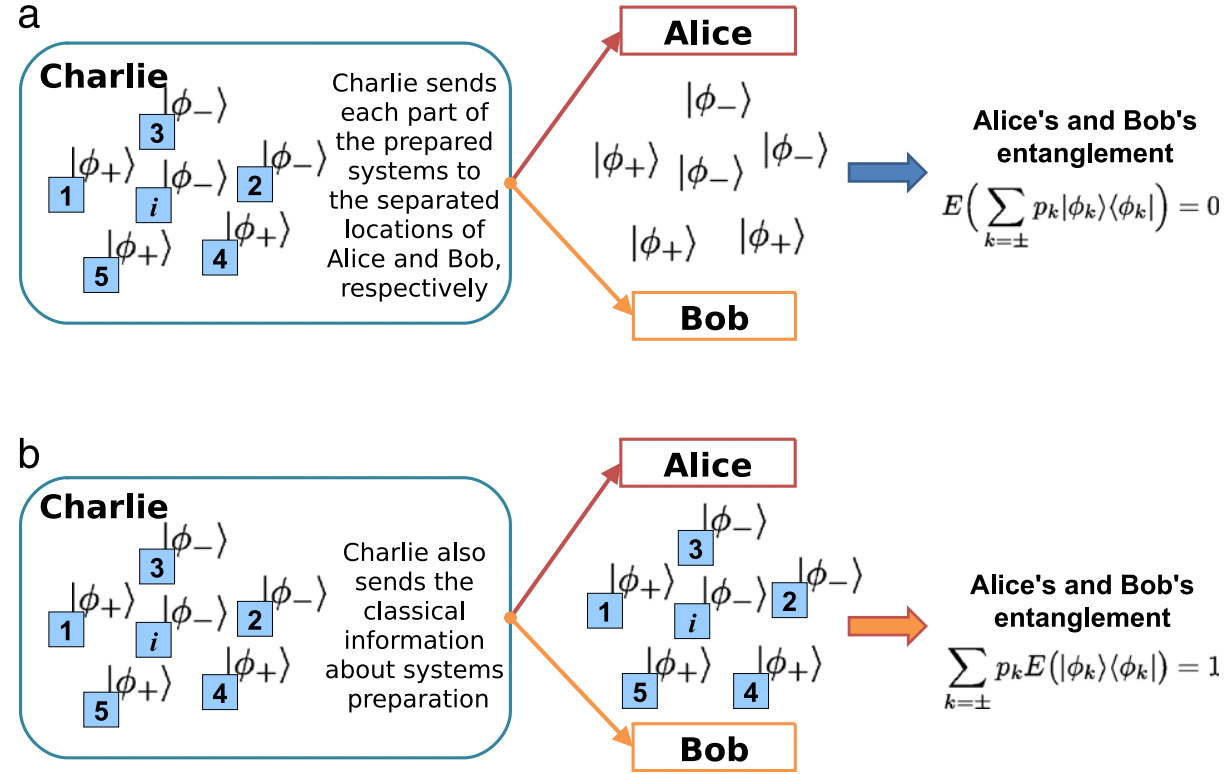

Fig. 2. (Color online) Pictorial illustration of the concept of hidden entanglement. Charlie prepares a large number of bipartite systems in the pure states $\left|\psi_{i}\right\rangle$, as described by the quantum ensemble $\mathcal{A}=\left\{\left(p_{i},\left|\psi_{i}\right\rangle\right)\right\}$. Here, for the sake of simplicity, we assume that $\left|\psi_{i}\right\rangle$ can be chosen as $\left|\phi_{ \pm}\right\rangle=(|00\rangle \pm|11\rangle) / \sqrt{2}$ with the same probability [12]. (a) Charlie sends one half of each system to Alice and the other half to Bob through noiseless quantum channels. The entanglement Alice and Bob can distil per pair vanishes, $E\left(\rho_{A B}\right)=0$, since Alice's and Bob's state $\rho_{A B}=\sum_{i} p_{i}\left|\psi_{i}\right\rangle\left\langle\psi_{i}\right|=\frac{1}{2}(|00\rangle\langle 00|+| 11\rangle\langle 11|)$ is separable. (b) Charlie uses a classical telephone line to communicate the states preparation to Alice. The entanglement Alice and Bob can now distil per pair is equal to 1 (Alice can perform a phase flip on her qubit, each time she knows that the corresponding pair is $\left|\phi_{-}\right\rangle$, so that all Alice's and Bob's pairs at the end are in the state $\left.\left|\phi_{+}\right\rangle\right)$. In the two scenarios, Alice and Bob physically share the same system. Here the root of entanglement recovery lies in the acquisition of classical information. Since this occurs in the absence of any interaction between the quantum systems or entanglement transfer through a third quantum system, the phenomenon is entirely due to the manifestation of quantum correlations already present in the system and in this sense "hidden".

admits a single physical decomposition in terms of an ensemble of pure state evolutions. This is always possible, at least in principle, when the system is affected by classical noise sources, as illustrated in Appendix A.1.

In the rest of this article we will illustrate the meaning of HE, expressed by Eq. (3), with various examples. There exist several inequivalent measures of mixed state entanglement [3,4]. Here we consider the entanglement of formation $E_{f}(\rho)$, which is an upper bound for any bipartite entanglement measure [13], so that $E_{\mathrm{av}}-E_{f}(\rho)$ is a lower bound for the hidden entanglement. $E_{f}(\rho)$ can be readily computed for two-qubit systems via the concurrence $C(\rho)[14]$.

\subsection{Entanglement revivals under random local fields}

We first illustrate the concept of HE by considering a random, local dynamics and demonstrating that, under proper conditions, a complete recovery of the entanglement $E_{f}(\rho)$ may occur.

A basic property of the average entanglement is its invariance under local unitary transformations. In particular this is the case of the evolution in a random local external field [15] inducing local random unitaries $U_{\alpha}(t) \otimes V_{\beta}(t)$ on a bipartite system, with the operators $U_{\alpha}$ and $V_{\beta}$ acting respectively on the first and on the second subsystem, and depending on the random variables $\alpha, \beta$. Let us suppose that the system is initially prepared in a pure state $\left|\varphi\left(t_{0}\right)\right\rangle=\left|\varphi_{0}\right\rangle$. Thus at any subsequent time the system is described by the quantum ensemble $\mathcal{A}(t)=\left\{\left(p_{\alpha \beta},\left|\varphi_{\alpha \beta}(t)\right\rangle\right)\right\}$, with $\left|\varphi_{\alpha \beta}(t)\right\rangle=\left(U_{\alpha}(t) \otimes V_{\beta}(t)\right)\left|\varphi_{0}\right\rangle$. The average entanglement of the ensemble $\mathcal{A}$ is conserved by this dynamics, $E_{\mathrm{av}}(\mathcal{A}(t))=E_{\mathrm{av}}\left(\mathcal{A}\left(t_{0}\right)\right)$. 
On the other hand, the entanglement of the mixture $\rho(t)=\sum_{\alpha, \beta} p_{\alpha \beta}\left|\varphi_{\alpha \beta}(t)\right\rangle\left\langle\varphi_{\alpha \beta}(t)\right|$ is only upper bounded by the average entanglement: $E_{f}(\rho(t)) \leq E_{\mathrm{av}}(\mathcal{A}(t))$, implying that a variable (time dependent) HE may exist. This is clearly illustrated by the following simple example.

Let us consider a two-qubit system $A B$ initially prepared in the maximally entangled Bell state $\left|\phi^{+}\right\rangle$. The time evolution consists of local unitaries, but we have no complete information about which local unitary is acting. In particular, we suppose that the qubit $A$ undergoes, with equal probability, a rotation about the $x$-axis of its Bloch sphere, $U_{x}(t)=\mathrm{e}^{-\mathrm{i} \sigma_{x} \omega t / 2}$, or a rotation around the $z$-axis, $U_{z}(t)=\mathrm{e}^{-\mathrm{i} \sigma_{z} \omega t / 2}$, while the qubit $B$ remains unchanged. Hence, the ensemble $\mathcal{A}$ at time $t$ is

$$
\mathcal{A}(t)=\left\{\left(\frac{1}{2},\left(U_{x}(t) \otimes \mathbb{1}_{B}\right)\left|\phi^{+}\right\rangle\right),\left(\frac{1}{2},\left(U_{z}(t) \otimes \mathbb{1}_{B}\right)\left|\phi^{+}\right\rangle\right)\right\} .
$$

Since we are dealing with random local unitaries, the average entanglement of $\mathcal{A}$ is constant in time, $E_{\mathrm{av}}(\mathcal{A}(t))=1$. On the other hand, the entanglement of the state $\rho(t)$ changes in time. At $\bar{t}=\frac{\pi}{\omega}$, $\rho(\bar{t})=\frac{1}{2}\left|\phi^{-}\right\rangle\left\langle\phi^{-}\left|+\frac{1}{2}\right| \psi^{+}\right\rangle\left\langle\psi^{+}\right|$is separable, whereas at $2 \bar{t}, U_{x}(2 \bar{t})=U_{z}(2 \bar{t})=\mathbb{1}_{A}$ and the initial maximally entangled state is recovered. ${ }^{2}$ In the interval $[\bar{t}, 2 \bar{t}]$ the entanglement revives from zero to one without the action of any nonlocal quantum operation, thus apparently violating the monotonicity axiom. The ensemble description tells us that at time $\bar{t}$ the system is always in an entangled state $\left(\left|\phi_{-}\right\rangle\right.$ or $\left.\left|\psi_{+}\right\rangle\right)$, but the lack of knowledge about which local operation the system underwent prevents us from distilling any entanglement: entanglement is hidden, $E_{h}(\mathcal{A}(\bar{t}))=1$ and $E_{f}(\rho(\bar{t}))=0$. At time $2 \bar{t}$ this lack of knowledge is irrelevant since the two possible time evolutions result in the identity operation $\mathbb{1}_{A}$ and entanglement is recovered, $E_{h}(\mathcal{A}(2 \bar{t}))=0$ and $E_{f}(\rho(2 \bar{t}))=1$.

We notice that entanglement revivals under random local fields have been studied in Ref. [5]. Here we explain and quantify this phenomenon in terms of HE.

\section{Entanglement recovery in the presence of classical non-Markovian noise}

A fingerprint of the existence of $\mathrm{HE}$ is the possibility to completely recover entanglement of a noisy bipartite system by the action of local pulses. Here we consider a simple system consisting of two noninteracting qubits affected by classical non-Markovian noise. This simplified model captures essential features of several nanodevices whose dynamics is dominated by low-frequency noise [16-19]. We suppose the two qubits are initially prepared in a Bell state $\left|\varphi_{0}\right\rangle$ and, for the sake of simplicity, assume that only qubit $A$ is affected by phase noise (pure dephasing), as described by $(\hbar=1)$

$$
\mathscr{H}_{A}(t)=\left[-\Omega_{A} \sigma_{z}+\varepsilon(t) \sigma_{z}+\mathcal{V}(t) \sigma_{x}\right] / 2,
$$

where $\varepsilon(t)$ is a stochastic process, and $\mathcal{V}(t)$ an external control field. Qubit $B$ evolves unitarily under a Hamiltonian $\mathscr{H}_{B}(t)$.

To start with, we suppose that $\varepsilon(t)$ is sufficiently slow to be considered static during the evolution time $t$, with a value randomly fluctuating from one quantum evolution to the other. We assume that $\varepsilon$ is a Gaussian random variable with zero expectation value and standard deviation $\sigma \cdot \mathcal{V}(t)$ indicates a hard echo $\pi$-pulse at time $\bar{t}$, short enough to neglect the effect of noise during its application. The evolution operator during the pulse is $\mathrm{e}^{-\mathrm{i} \sigma_{\chi} \pi / 2}=-\mathrm{i} \sigma_{x}$. Static noise [16-19] produces an effect analogous to inhomogeneous broadening in nuclear magnetic resonance (NMR) [20]. The system dynamics is described by the quantum ensemble $\mathcal{A}(t)=\left\{p(\varepsilon) d \varepsilon,\left|\varphi_{\varepsilon}(t)\right\rangle\right\}$, where $\left|\varphi_{\varepsilon}(t)\right\rangle=$ $\hat{T} \mathrm{e}^{-\mathrm{i} \int_{0}^{t} \mathscr{H}_{A}\left(t^{\prime}\right) d t^{\prime}} \otimes \hat{T} \mathrm{e}^{-\mathrm{i} \int_{0}^{t} \mathscr{H}_{B}\left(t^{\prime}\right) d t^{\prime}}\left|\varphi_{0}\right\rangle$ and $p(\varepsilon)$ is the Gaussian probability density function of $\varepsilon$ (see Appendix A.1). Note that for each realization of the stochastic process $\varepsilon(t)$ the system state acquires a random phase. In the density operator description of the system, the information about such random phase is lost by averaging the evolved pure state $\left|\varphi_{\varepsilon}(t)\right\rangle$ with respect to the random variable $\varepsilon$ :

\footnotetext{
2 We use the notation $\left|\psi^{ \pm}\right\rangle=(|01\rangle \pm|10\rangle) / \sqrt{2}$.
} 
$\rho(t)=\int d \varepsilon p(\varepsilon)\left|\varphi_{\varepsilon}(t)\right\rangle\left\langle\varphi_{\varepsilon}(t)\right|$. The addition of the local pulse at $\bar{t}$ produces a gradual cancellation of the random phase for each realization:

$$
\begin{aligned}
\left|\varphi_{\varepsilon}(t>\bar{t})\right\rangle & =-\mathrm{i} \mathrm{e}^{-\frac{\Omega_{A}+\varepsilon}{2} \sigma_{z}(t-\bar{t})} \sigma_{x} \mathrm{e}^{-\frac{\Omega_{A}+\varepsilon}{2} \sigma_{z} \bar{t}} \otimes \hat{T} \mathrm{e}^{-\mathrm{i} \int_{0}^{t} \mathscr{H}_{B}\left(t^{\prime}\right) d t^{\prime}}\left|\varphi_{0}\right\rangle \\
& =-\mathrm{i} \mathrm{e}^{-\frac{\Omega_{A}+\varepsilon}{2} \sigma_{z}(t-2 \bar{t})} \sigma_{x} \otimes \hat{T} \mathrm{e}^{-\mathrm{i} \int_{0}^{t} \not \mathcal{H}_{B}\left(t^{\prime}\right) d t^{\prime}}\left|\varphi_{0}\right\rangle,
\end{aligned}
$$

so that at time $t=2 \bar{t}$ the effect of the random phase is vanishing, also on the average state $\rho(t)$. A system prepared in a Bell state $\left|\varphi_{0}\right\rangle$ evolves in a mixture whose concurrence $C(\rho(t))$ is twice the absolute value of the only non-zero coherences, and reads

$$
C(\rho(t))= \begin{cases}\mathrm{e}^{-\frac{1}{2} \sigma^{2} t^{2}}, & 0 \leq t \leq \bar{t} \\ \mathrm{e}^{-\frac{1}{2} \sigma^{2}(t-2 \bar{t})^{2}}, & \bar{t} \leq t \leq 2 \bar{t} .\end{cases}
$$

The entanglement of formation $E_{f}(\rho(t))$ is obtained directly from $C(\rho(t))$ [14]:

$$
E_{f}(\rho(t))=\mathrm{h}\left(\frac{1+\sqrt{1-C(\rho(t))^{2}}}{2}\right),
$$

where $\mathrm{h}(x)=-x \log _{2} x-(1-x) \log _{2}(1-x)$. In the absence of pulses, $E_{f}$ decays and almost vanishes, $E_{f}(\rho(t)) \simeq 0$, at times $\sigma t \gg 1$, see Fig. 3, top panel, thick (red) curve. Differently, after the action of a local pulse at $t=\bar{t}$ the entanglement increases reaching at $t=2 \bar{t}$ its initial maximum value $E_{f}(\rho(2 \bar{t}))=E_{f}^{\max }=1$ (thin blue curve). This is exactly the average entanglement (dashed line) of the evolved physical ensemble $\mathcal{A}, E_{\mathrm{av}}(\mathcal{A}(t))=1$. This is reminiscent of Fig. 2 . For each realization of $\varepsilon(t)$, the system remains in a pure maximally entangled state. Entanglement degradation is due to a lack of classical knowledge on the system $A B$, namely on the random phase $\varepsilon$. Here, the classical information needed to recover entanglement, is de facto retrieved by the local pulse. The evolution after the pulse corresponds, in the example of Fig. 2, to the action of Alice next to the classical information received from Charlie.

In the general case of a Gaussian stochastic process $\varepsilon(t)$ the concurrence can be expressed in the form $[18,21]$

$$
C(\rho(t))=\mathrm{e}^{-\frac{1}{2} \int_{-\infty}^{+\infty} \frac{d \omega}{2 \pi} S(\omega) \frac{F(\omega, t)}{\omega^{2}}}
$$

where $S(\omega)=\int_{-\infty}^{+\infty} d t \mathrm{e}^{-\mathrm{i} \omega t}\langle\varepsilon(t) \varepsilon(0)\rangle$ is the power spectrum of the process $\varepsilon(t)$. The function $F(\omega, t)$ represents a filter function [18,21] depending on the system unitary evolution. When the system freely evolves under $\mathscr{H}_{A}(t)$ in the absence of external control actions $(\mathcal{V}(t)=0$ in (5)), the filter function $F_{\text {free }}(\omega, t)$ reads

$$
F_{\text {free }}(\omega, t)=4 \sin ^{2}\left(\frac{\omega t}{2}\right) .
$$

For an echo protocol, with a $\pi$-pulse at time $\bar{t}$ the filter function reads [17]

$$
F_{\text {echo }}(\omega, t)=4\left[\sin ^{2} \frac{\omega \bar{t}}{2}+\sin ^{2} \frac{\omega(t-\bar{t})}{2}-2 \cos \frac{\omega t}{2} \sin \frac{\omega \bar{t}}{2} \sin \frac{\omega(t-\bar{t})}{2}\right] .
$$

We consider a stochastic process with an exponential autocorrelation function, $\langle\varepsilon(t) \varepsilon(0)\rangle=\sigma^{2}$ $\mathrm{e}^{-|t| / \tau}$, with noise correlation time $\tau$. Also in this case, a local echo pulse leads to a significant entanglement recovery provided that the noise correlation time is sufficiently large, $\tau \gg \bar{t}$ (Fig. 3, top panel, dotted curves). Better performances can be achieved applying a train of pulses. For a periodic dynamical decoupling (PDD) protocol, i.e. a sequence of $\pi$-pulses applied at equally spaced times $t_{k}=k \Delta t$, the concurrence takes the form (9) with the filter function [22]

$$
F_{\text {PDD }}^{(\Delta t)}(\omega, t)=\left|1+(-1)^{\bar{n}} e^{\mathrm{i} \omega t}+2 \sum_{k=1}^{\bar{n}}(-1)^{k} e^{\mathrm{i} \omega k \Delta t}\right|^{2},
$$



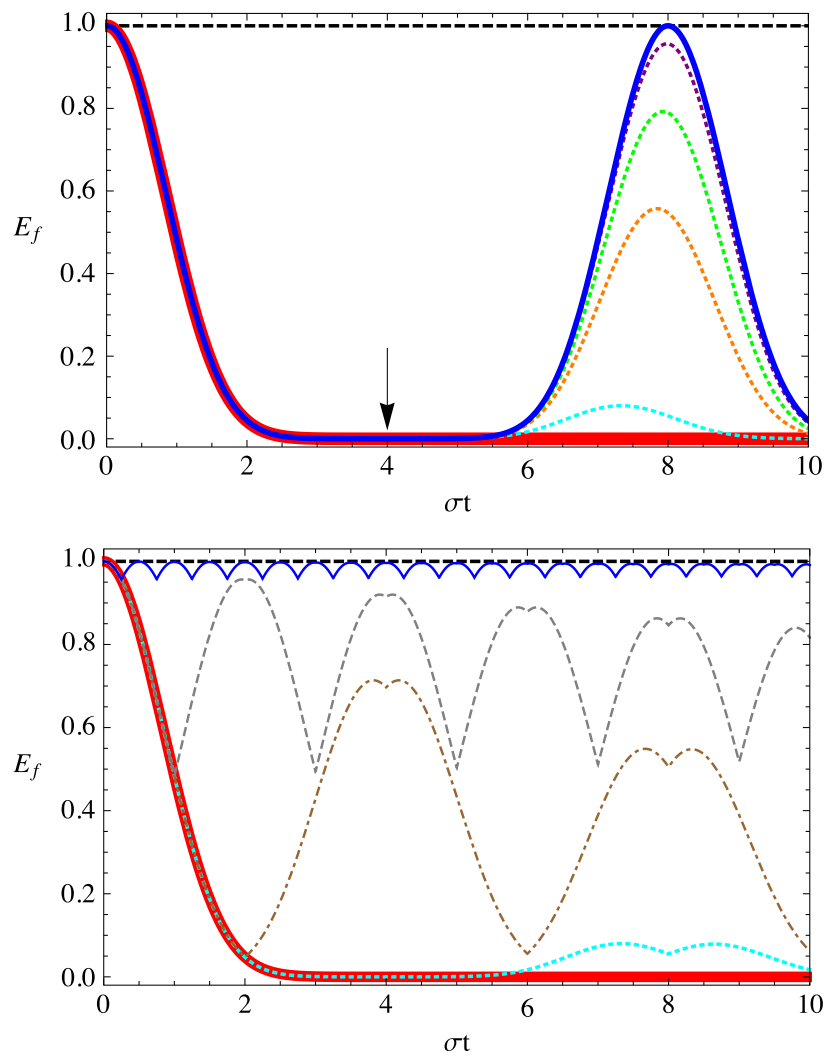

Fig. 3. Entanglement of formation $E_{f}(\rho(t))$ as a function of the dimensionless time $\sigma t$. Top panel: The thick (red) curve corresponds to the free evolution in the presence of static noise, the thin (blue) solid curve is the result of the echo pulse applied at time $\sigma \bar{t}=4$ (indicated by the arrow), Eq. (7). The dashed line is the system average entanglement $E_{\mathrm{av}}(\mathcal{A}(t))=1$. Dotted curves represent $E_{f}(\rho(t))$ for a $\varepsilon(t)$ with a Lorentzian power spectrum when an echo pulse is applied at time $\sigma \bar{t}=4$, from Eqs. (9) and (11). From bottom to top: $\sigma \tau=20$ (cyan curve), 100 (orange curve), 200 (green curve), and 500 (purple curve). Perfect recovery is obtained in the limit $\tau / \bar{t} \rightarrow \infty$, corresponding to static noise (blue thin solid curve). Bottom panel: The (red) thick solid curve corresponds to $E_{f}(\rho(t))$ evaluated for a stochastic process $\varepsilon(t)$ with a Lorentzian power spectrum and correlation time $\sigma \tau=20$, in the case of free evolution, from Eqs. (9) and (10). The other curves refer to a PDD protocol applied to qubit A with equally spaced $\pi$-pulses, applied at times $t_{k}=k \Delta t$. $E_{f}(\rho(t))$ is numerically evaluated from Eqs. (9) and (12): $\tau / \Delta t=5$ for the dotted (cyan) curve, $\tau / \Delta t=10$ for the dot-dashed (brown) curve, $\tau / \Delta t=20$ for the dashed (gray) curve and $\tau / \Delta t=80$ for the thin solid (blue) curve. Almost perfect recovery is obtained when $\tau / \Delta t \gg 1$. (For interpretation of the references to color in this figure legend, the reader is referred to the web version of this article.)

where $\bar{n}$ denotes the integer part of $\frac{t}{\Delta t}$. In this case, the recovery improves with increasing the ratio $\tau / \Delta t$ between the noise correlation time and the time interval between consecutive pulses, see Fig. 3 , bottom panel.

These examples show the possibility to fully recover the entanglement $E(\rho)$ by a local operation. The physical mechanism behind this phenomenon is very simple: a local $\pi$-pulse applied at some time $\bar{t}$ refocuses the different qubit quantum evolutions restoring at time $2 \bar{t}$ the qubit $A$ coherence and consequently (qubit $B$ evolves unitarily) causing the entanglement to reappear, with an efficiency depending on the correlation time of the stochastic process. Note that the non-Markovian nature of the stochastic process is a necessary (but not sufficient) condition for the non-monotonous entanglement behavior. Indeed, to observe revivals the environment must keep memory of its states on a time scale larger than the system evolution time. Under this condition, after the pulse there is a "back-flow" [23] of the classical information on the system's phase which the environment has acquired during the evolution before the pulse, see Appendix A.1.1. 
a

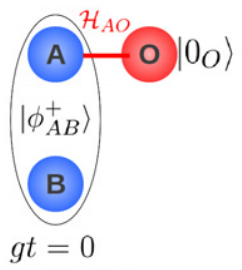

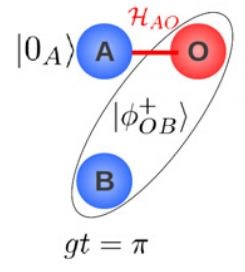

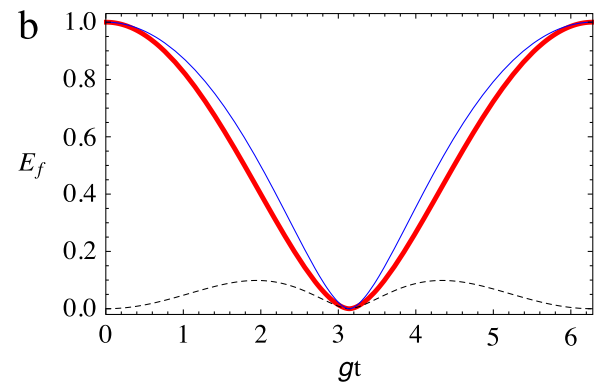

Fig. 4. Panel (a): Two qubits are initially $(g t=0)$ prepared in the Bell state $\left|\phi_{A B}^{+}\right\rangle=(|00\rangle+|11\rangle) / \sqrt{2}$. Qubit $B$ is virtually isolated from any environment. Qubit $A$ resonantly interacts with a harmonic oscillator $O$ via a Jaynes-Cummings Hamiltonian. The oscillator is initially in its ground state $\left|0_{O}\right\rangle$. At time $g t=\pi$, because of the interaction between $A$ and $O$, the states of the two systems are swapped. Panel (b): Entanglement of formation $E_{f}\left(\rho_{A B}(t)\right)$ (thick red line), average entanglement $E_{\mathrm{av}}(\mathcal{A}(t))$ from Eq. (15) relative to $A B$ quantum ensemble Eqs. (13)-(14) (thin blue line) and corresponding hidden entanglement Eq. (16) (dashed line) as a function of the dimensionless time $g t$. The vanishing $A B$ entanglement at $g t=\pi$ is due to entanglement transfer to the $B O$ system, not to the lack of any classical information on the system $A B$. (For interpretation of the references to color in this figure legend, the reader is referred to the web version of this article.)

Entanglement revival after the application of the pulses may appear paradoxical at first sight. Entanglement is by definition a nonlocal resource, whereas we only acted locally on one qubit, without any transfer of entanglement from the environment which is a classical noise source. The key point is that here entanglement is not destroyed during the time evolution, as indicated by the average entanglement of the ensemble $\mathcal{A}$ describing the system dynamics, which is maximum at any time $E_{\mathrm{av}}(\mathcal{A}(t))=1$. Entanglement is instead hidden: because of the lack of classical knowledge on the system state due to defocusing among the different evolutions of the (maximally entangled) states of $\mathcal{A}(t)$, the HE grows before the pulse is applied. After the pulse, this lack of classical knowledge is gradually reduced, vanishing in the limit $\tau / \bar{t} \rightarrow \infty$ (echo) or $\tau / \Delta t \rightarrow \infty$ (PDD). This is the reason why entanglement can be recovered without any nonlocal control.

\subsection{Nature of entanglement revivals}

The phenomenon of entanglement revivals we have examined is conceptually different from the revivals that a system can exhibit due to the interaction with a non-Markovian quantum environment. Indeed, in this last case, system and environment can also develop quantum correlations, and entanglement revivals may originate from a different physical mechanism. To exemplify the conceptual difference between these two situations here we consider a fully quantum system where the entanglement dynamics cyclically decreases, vanishes at a time $\bar{t}$ and then increases, analogously to the case of two qubits in random local fields.

Let us consider a two-qubit system $A-B$ where $A$ resonantly interacts with a quantum harmonic oscillator $O$ via a Jaynes-Cummings (JC) Hamiltonian, an assumption frequently performed in cavity quantum electrodynamics (QED) (see, for instance, [24,25]). Qubit $B$ is virtually isolated from any environment. Initially, the two qubits are prepared in the Bell state $\left|\phi_{A B}^{+}\right\rangle$and $O$ in its ground state $\left|0_{O}\right\rangle$, see Fig. 4(a) left. In the interaction picture, the Hamiltonian is $\mathscr{H}_{A O}=g\left(\sigma_{+} a+\sigma_{-} a^{\dagger}\right)$, where $g$ is the coupling constant, $\sigma_{+}\left(\sigma_{-}\right)$the qubit raising (lowering) operator, and $a^{\dagger}(a)$ the oscillator creation (annihilation) operator. At time $\bar{t}=\pi / g$ the states of $A$ and $O$ are swapped with respect to the initial state, and the global state becomes $\left|0_{A}\right\rangle \otimes\left|\phi_{B 0}^{+}\right\rangle$, see Fig. 4(a) right. We have that $\rho_{A B}(\bar{t})=\left|0_{A}\right\rangle \otimes \frac{1}{2} \mathbb{1}_{B}$, the $A-B$ entanglement is zero, being completely transferred to $B-O$. At time $\bar{t}$, any unraveling of the $A-B$ dynamics (see Appendix A.2) gives a quantum ensemble whose average entanglement is zero, so that $E_{h}(\bar{t})=0$ : at this time no classical communication or local operation can help to recover any entanglement between $A$ and $B$. Only the subsequent interaction between $A$ and $O$ can gradually restore the $A-B$ entanglement: At time $2 \bar{t}$, when a new $A-O$ swapping is completed, the initial state is just retrieved. Therefore, the entanglement revival is here due to the perfect entanglement backtransfer, as well-known in the literature [26,27]. 
This explanation is unsuitable for the examples considered above in this paper, where the environment is classical and no entanglement transfer is possible. In those cases, during the dynamics, the environment acquires only classical information about the system $A-B$ and does not entangle with $A$ or $B$. Quantum correlations do not leave the system $A-B$ but they are simply not accessible due to the lack of classical information. Indeed, if at time $\bar{t}$ when $E_{h}(\bar{t})=1$, someone provides $A-B$ with the classical information about which random unitary the system underwent (in the case of random local fields) or about which random phase is added to the system states (in the case of stochastic pure-dephasing noise), then all the $A-B$ entanglement can be recovered.

In order to get further insight on the phenomenon occurring in the quantum system $A B O$, here we estimate the hidden entanglement between $A$ and $B$. To this end we suppose to perform a measurement of the system $O$ in the orthonormal basis $\{|0\rangle,|1\rangle\} .{ }^{3}$ Under these conditions the physical quantum ensemble describing the $A B$ quantum dynamics reads $\mathcal{A}=\left\{\left(p_{0}(t),\left|\varphi_{0}(t)\right\rangle\right),\left(p_{1}(t),\left|\varphi_{1}(t)\right\rangle\right)\right\}$, where

$$
p_{0}(t)=\frac{1}{2}\left(1+\cos ^{2}(g t / 2)\right), \quad p_{1}(t)=\frac{1}{2} \sin ^{2}(g t / 2)
$$

are respectively the probability that the system $O$ is found in $|0\rangle$ or in $|1\rangle$, and

$$
\left|\varphi_{0}(t)\right\rangle=\frac{1}{\sqrt{2 p_{0}(t)}}(|00\rangle+\cos (g t / 2)|11\rangle), \quad\left|\varphi_{1}(t)\right\rangle=|01\rangle
$$

are the corresponding system $A B$ states. Note that only the state $\left|\varphi_{0}(t)\right\rangle$ is an entangled state. Therefore the average entanglement of the quantum ensemble $\mathcal{A}$ is given by:

$$
E_{\mathrm{av}}(\mathcal{A}, t)=p_{0}(t) E\left(\left|\varphi_{0}(t)\right\rangle\left\langle\varphi_{0}(t)\right|\right)=\frac{1+\eta}{2} \mathrm{f}\left(\frac{2 \sqrt{\eta}}{1+\eta}\right)
$$

and the hidden entanglement reads

$$
E_{h}(\mathcal{A}, t)=E_{f}\left(\rho_{A B}(t)\right)-E_{\mathrm{av}}(\mathcal{A}, t)=\mathrm{f}(\sqrt{\eta})-\frac{1+\eta}{2} \mathrm{f}\left(\frac{2 \sqrt{\eta}}{1+\eta}\right),
$$

where we set $f(x) \equiv h\left(\frac{1+\sqrt{1-x^{2}}}{2}\right)$, with the function $h$ defined below Eq. (8), and $\eta=\cos ^{2}(g t / 2)$. In Fig. $4(\mathrm{~b})$ we show $E_{\mathrm{av}}(\mathcal{A}, t)$ (thin blue line), the entanglement of formation $E_{f}\left(\rho_{A B}(t)\right)$ (thick red line) and the corresponding hidden entanglement $E_{h}(\mathcal{A}(t))$ (dashed line). ${ }^{4}$ We observe the existence of a small amount of $\mathrm{HE}$ at times $g t \neq \pi$. It represents the extra (with respect to the entanglement of formation) amount of entanglement that it would be possible to recover if the classical information coming from measurements of the quantum state of $O$ would be available. The fact that $E_{h}(\mathcal{A}(t))$ is much smaller than the initially present entanglement indicates that the main mechanism underlying the decrease (recover) of $A B$ entanglement, it is not the loss (gain) of classical information on the system $A B$, but it is rather the development (regression) of quantum correlations between $B$ and $O$.

A few remarks are now in order. Similar entanglement revivals can be observed when the quantum harmonic oscillator interacts with an environment inducing a non-Markovian dynamics of $A-B(O$ plus its environment representing a structured bath acting locally on $A$ and influencing nontrivially the quantum evolution of $A B$ ). In this case, the entanglement recovery signals the non-Markovian quantum evolution of one of the parts of the bipartite system $A B$ acted by a quantum environment, as discussed in Ref. [29]. The example of the two qubits affected by low-frequency noise highlights that when a bipartite quantum system is affected by classical non-Markovian noise, the induced nonMarkovian dynamics of the bipartite system does not justify by itself the occurrence of entanglement

\footnotetext{
3 The physical decomposition of the $A B$ system interacting with another quantum system in general is not unique, see Appendix A.2. The decomposition we choose may be physically realizable in cavity QED systems, moreover one can numerically check that it gives the largest amount of average entanglement [28].

4 It is worth to notice that Eq. (16) also gives the hidden entanglement associable to an amplitude damping channel [1,2] applied to the qubit $A$, where $1-\eta$ is the probability that $A$ loses a photon.
} 
revivals between the independent subsystems. Indeed, in Fig. 3 the dynamics without $\pi$-pulses is non-Markovian and entanglement revivals do not appear. We may summarize the above observations saying that the origin of entanglement revivals displayed by a noninteracting bipartite quantum system may be either due to (i) a back-and-forth transfer of quantum correlations with a quantum environment possibly acting locally on one of the two subsystems and inducing a non-Markovian dynamics, or to (ii) the action of local operations on one subsystem affected by a classical nonMarkovian noise source. In this last case the recovery is just the manifestation of quantum correlations which remain "hidden" in the quantum system, i.e. not directly available in the density matrix description of the system state.

\section{Discussion}

From the examples above we argue that recovery of entanglement is achievable without nonlocal operations, when various members of the physical ensemble evolve differently from each other but unitarily. In this case, even though the evolution of the ensemble averaged density matrix is not unitary, no qubit-environment entanglement is generated. Using a terminology borrowed from NMR [30] we speak of incoherent errors, whereas decoherent errors arise when the evolution is nonunitary even for a single member of the ensemble. For this latter case the average entanglement decays.

We point out that HE depends on the quantum ensemble physically giving the system state. The key point is that the physical dynamics subsumes a specific decomposition for the evolved density matrix of the system. In the above examples relative to random local fields and classical non-Markovian noise, such physical decomposition is always an ensemble of maximally entangled states. Had we considered a dynamics such as to give, at $t=\bar{t}$, a mixture of separable states as decomposition of the system density matrix, no local operation would have been capable to recover entanglement $\left(E_{h}(\bar{t})=0\right)$. The dynamics of the system after the application of the pulse proves that, at time $\bar{t}$, the two decompositions are not equivalent.

Finally, we remark that the results of the previous examples do not violate the monotonicity axiom [3,4,7]: entanglement cannot increase under LOCC. The key point is that this axiom is fulfilled by all entanglement measures provided we consider local operations which are completely positive trace preserving (CPT) maps. On the other hand, not all physical operations result in a composition of completely positive trace preserving maps across successive time intervals: there exist non-divisible dynamical maps, as discussed in Refs. [23,29]. In the previous examples entanglement recovery, from time $\bar{t}$ to time $2 \bar{t}$, is induced by purely local operations which are not LOCC, since the corresponding density matrix evolution cannot be described by a CPT map. To prove this point, it is enough to observe that the density matrix is such that $\rho(2 \bar{t})=\rho(0)$. Therefore driving the system from the state $\rho(\bar{t})$ to $\rho(2 \bar{t})$ is equivalent to driving it to $\rho(0)$. This operation cannot be described by a CPT map since the (CPT) evolution from time 0 to time $\bar{t}$ is not invertible.

\section{Conclusions}

In conclusion, we have introduced the concept of hidden entanglement (HE) based on the description of the system dynamics in terms of the ensemble of pure states physically underlying the system time evolution. We used the concept of HE to give a physical explanation on the phenomenon of entanglement revivals, in those cases in which the system is subject to classical noise sources. We showed as there is no violation of the entanglement monotonicity axiom because no entanglement is destroyed and created in this case: a nonzero HE signals a loss of entanglement that is not due to the establishment of quantum correlations with the environment. Quantum correlations remain within the system, but they are not exploitable due to the lack of classical information.

The concept of HE can be also applied when a system interacts with a quantum environment. In these cases, however, evaluation of HE requires some environment "monitoring strategy" $[9,10,28$, 31-35], in order to realize a statistical ensemble of pure state evolutions which physically underlies the system dynamics. 
We stress that our analysis has direct application to solid state nanodevices which prevalently suffer from low-frequency noise. Indeed HE may be a figure of merit indicating the amount of entanglement resources which can be recovered by using local sequences of standard pulses, an appealing feature for quantum control in distributed architectures for quantum computing where different subunits are subject to different non-Markovian noise sources. This allows us to avoid resorting to non-local control [36], which may be a much more demanding task.

\section{Acknowledgments}

A.D. thanks Laura Mazzola for useful discussions. R.L.F. thanks Giuseppe Compagno for fruitful discussions. This work was partially supported by the European Community through grant no. ITN-2008-234970 NANOCTM and by PON02-00355-339123-ENERGETIC. G.B. acknowledges support by MIUR-PRIN project Collective quantum phenomena: From strongly correlated systems to quantum simulators.

\section{Appendix. Quantum ensembles and system dynamics}

\section{A.1. Evolution in the presence of classical noise}

In this appendix we demonstrate that when a system is affected by classical noise sources its dynamics admits a single physical decomposition in terms of an ensemble of pure state evolutions. To this end let us consider a quantum system $Q$ that evolves according to the following Hamiltonian:

$$
\mathscr{H}_{Q}(t)=\mathscr{H}_{0}+\hat{q} x(t),
$$

where $\mathscr{H}$ and $\hat{q}$ are Hermitian operators acting on the system's Hilbert space, and $x(t)$ is a stochastic process representing the effect of a classical noise source. The system quantum evolution for a given realization of $x(t)$ is expressed in terms of the evolution operator

$$
U_{Q}(t \mid x(t))=\hat{T} \mathrm{e}^{-\mathrm{i} \int_{0}^{t} d t^{\prime} \mathscr{H}_{Q}\left(t^{\prime}\right)} .
$$

Assuming that the system is initially prepared in a pure state $|\psi(t=0)\rangle=\left|\psi_{0}\right\rangle$, the system state at a generic time $t$ is

$$
|\psi(t \mid x(t))\rangle=U_{Q}(t \mid x(t))\left|\psi_{0}\right\rangle
$$

The system evolution is represented by the quantum ensemble

$$
\mathcal{A}=\{P[x(t)],|\psi(t \mid x(t))\rangle\}
$$

where $P[x(t)]$ is the probability of a given realization $x(t)$. The system density matrix is obtained by averaging over the quantum ensemble (A.4), and it is expressed as a path integral:

$$
\rho(t)=\int D[x(t)] P[x(t)]|\psi(t \mid x(t))\rangle\langle\psi(t \mid x(t))| .
$$

This description applies to several scenarios in the solid state [20,37]. In relevant situations the stochastic process $x(t)$ can be considered static during the time evolution (see for instance [16]). In these cases $x(t)$ can be replaced by a random variable $x$ and the probability $P[x(t)]$ is replaced with the probability density function $p(x)$. The path-integral (A.5) reduces to an ordinary integral:

$$
\rho(t)=\int d x p(x)\left|\psi_{x}(t)\right\rangle\left\langle\psi_{x}(t)\right|, \quad \mathcal{A}=\left\{p(x) d x,\left|\psi_{x}(t)\right\rangle\right\},
$$

where $\left|\psi_{x}(t)\right\rangle=|\psi(t \mid x)\rangle$. If $x$ is a discrete random variable the quantum ensemble reduces to the general form introduced in Section 2

$$
\mathcal{A}=\left\{p_{i},\left|\psi_{i}(t)\right\rangle\right\}, \quad \rho(t)=\sum_{i} p_{i}\left|\psi_{i}(t)\right\rangle\left\langle\psi_{i}(t)\right|,
$$

where $p_{i} \equiv p\left(x=x_{i}\right)$, and $\left|\psi_{i}(t)\right\rangle=\left|\psi\left(t \mid x_{i}\right)\right\rangle$. 


\section{A.1.1. System-environment information flow}

In this appendix we outline the relationship between hidden entanglement recovery and backflow of classical information from the environment to the system (see also Ref. [38]). We start by considering the quantum ensemble $\mathcal{A}(t)$ of Eq. (A.7) and embed the randomness inherent this quantum ensemble into the degrees of freedom of a dummy quantum system $E[12,39]$, in the following way:

$$
\begin{aligned}
& \rho(t)=\rho^{S}(t)=\operatorname{Tr}_{E}\left[\rho^{S E}(t)\right], \\
& \rho^{S E}(t)=\sum_{i} p_{i}\left|x_{i}\right\rangle\left\langle x_{i}|\otimes| \psi_{i}(t)\right\rangle\left\langle\psi_{i}(t)\right|,
\end{aligned}
$$

where $\left\{\left|x_{i}\right\rangle\right\}$ is an orthonormal basis for $E$. From (A.9) one can compute the quantum mutual information $[1,2]$ between the fictitious environment $E$ and the system $S$ :

$$
I(S: E)=S(\rho(t))+S\left(\rho^{E}(t)\right)-S\left(\rho^{S E}(t)\right)
$$

where $\rho^{E}(t)=\operatorname{Tr}_{S}\left[\rho^{S E}(t)\right]$. Using the relation $S\left(\sum_{i} p_{i}\left|x_{i}\right\rangle\left\langle x_{i}\right| \otimes \rho_{i}\right)=H\left(p_{i}\right)+\sum_{i} p_{i} S\left(\rho_{i}\right)$ [1], we obtain

$$
I(S: E)=S(\rho(t))+H\left(p_{i}\right)-H\left(p_{i}\right)=S(\rho(t)),
$$

where $H\left(p_{i}\right)=-\sum_{i} p_{i} \log _{2} p_{i}$ is the Shannon entropy associated with the probability distribution $\left\{p_{i}\right\}$. Therefore, the loss of knowledge on the system can be explained as due to the correlations developed between $S$ and $E$. We can describe the information flow [23] between $S$ and $E$ by the quantum mutual information $I(S: E)$ [40,41], a backward flow occurring when $\partial I(S: E) / \partial t<0$ [40]. Note that the above discussion can be easily extended to the case of a continuous random variable $x$ rather than a discrete one $x_{i}$.

In the example of Fig. 3 (top), $I(S: E)$ exhibits a behavior qualitatively and quantitatively close to that of $1-E_{f}$, the back-flow of information just occurring in the same interval in which entanglement turns to increase.

\section{A.2. Evolution in the presence of a quantum environment}

The description in terms of quantum ensemble we use in Appendix A.1 is no longer unique when the quantum system interacts with a quantum environment. To illustrate this fact let us suppose that the quantum system is coupled to a quantum environment $E$ with a total Hamiltonian for $Q$ and $E$ given by

$$
\mathscr{H}_{\mathrm{QE}}=\mathscr{H}_{0}+\hat{q} \otimes \hat{X}_{E}+\mathscr{H}_{E}
$$

where $\mathscr{H}_{0}$ and $\hat{q}$ are the same system $Q$ operators which appear in (A.1), $\hat{X}_{E}$ is an Hermitian operator for the environment $E$ and $\mathscr{H}_{E}$ is free evolution Hamiltonian for the environment. The system-plusenvironment evolves unitarily with

$$
U_{Q E}(t)=\mathrm{e}^{-\mathrm{i} \mathscr{H}_{Q E} t}
$$

For the sake of simplicity here we assume that the system $Q E$ is initially in the state $\left|\Psi_{Q E}(0)\right\rangle=$ $\left|\psi_{0}\right\rangle \otimes\left|\xi_{E}\right\rangle[1,2]$. The system $Q E$ state at time $t$ is:

$$
\left|\Psi_{Q E}(t)\right\rangle=U_{Q E}(t)\left|\psi_{0}\right\rangle \otimes\left|\xi_{E}\right\rangle
$$

The reduced density matrix of $Q$ is

$$
\rho_{Q}=\operatorname{Tr}_{E}\left\{U_{Q E}(t)\left|\psi_{0}\right\rangle\left\langle\psi_{0}|\otimes| \xi_{E}\right\rangle\left\langle\xi_{E}\right| U_{Q E}^{\dagger}(t)\right\}
$$


The trace operation on the environment $E$ can be carried out by choosing an orthonormal basis $\kappa=\left\{\left|k_{E}\right\rangle\right\}$ for $E$ :

$$
\begin{aligned}
\rho_{Q} & =\sum_{k}\left\langle k_{E}\left|\left(U_{Q E}(t)\left|\psi_{0}\right\rangle\left\langle\psi_{0}|\otimes| \xi_{E}\right\rangle\left\langle\xi_{E}\right| U_{Q E}^{\dagger}(t)\right)\right| k_{E}\right\rangle \\
& =\sum_{k}\left\langle k_{E}\left|U_{Q E}(t)\right| \xi_{E}\right\rangle\left|\psi_{0}\right\rangle\left\langle\psi_{0}\right|\left\langle\xi_{E}\left|U_{Q E}^{\dagger}(t)\right| k_{E}\right\rangle \\
& =\sum_{k} U_{Q_{k}}(t)\left|\psi_{0}\right\rangle\left\langle\psi_{0}\right| U_{Q_{k}}^{\dagger}(t),
\end{aligned}
$$

where $U_{Q_{k}}(t) \equiv\left\langle k_{E}\left|U_{Q E}(t)\right| \xi_{E}\right\rangle$ are system $Q$ operators. Note that in general $U_{Q_{k}}(t)$ are not unitary operators. By defining the normalized $Q$ state

$$
\left|\psi_{k}(t)\right\rangle=\frac{1}{\sqrt{\eta_{k}}} U_{Q_{k}}(t)\left|\psi_{0}\right\rangle
$$

where

$$
\eta_{k}=\left\langle\psi_{0}\left|U_{Q_{k}}^{\dagger}(t) U_{Q_{k}}(t)\right| \psi_{0}\right\rangle,
$$

the reduced density matrix for $Q$ reads

$$
\rho_{Q}(t)=\sum_{k} \eta_{k}\left|\psi_{k}(t)\right\rangle\left\langle\psi_{k}(t)\right|
$$

The trace operation corresponds to perform a measurement of the system $E$ with respect to the orthonormal basis $\kappa=\left\{\left|k_{E}\right\rangle\right\}$. When the outcome of the measurement is $k$ then the system $Q$ is in the pure state $\left|\psi_{k}(t)\right\rangle$. Therefore, the system $Q$ is described by the quantum ensemble

$$
\mathcal{A}_{\kappa}=\left\{\eta_{k},\left|\psi_{k}(t)\right\rangle\right\},
$$

where $\eta_{k}$ is the probability to obtain the outcome $k$, given by (A.18). We have unraveled the $Q$ dynamics into a statistical ensemble of pure state evolutions, the so-called quantum trajectories [42].

The measurement of the environment $E$ removes the arbitrariness of the $\rho_{Q}$ decomposition, the system $Q$ being physically described $[9,10,28,33,43]$ by the ensemble (A.20). Once the classical information about the outcomes $k$ is known, the average entanglement $E_{\text {av }}\left(\mathcal{A}_{k}\right)$ can be obtained by means of local operations. Also in this case, hidden entanglement represents the amount of entanglement which can be recovered, once the classical information is provided to the system. We remark that in general the above information about the environment is not easily accessible, since the environment is supposed to represent an ensemble of a huge number of uncontrollable degrees of freedom. Nevertheless, under limiting situations it may be possible to have some control on a selection of those degrees of freedom [35].

The above considerations also point out that when a system interacts with a quantum environment, the average entanglement (and consequently the hidden entanglement) depends on the adopted environment monitoring strategy. One can in principle look for the system ensemble with the largest amount of average entanglement; this maximum entanglement is called entanglement of assistance [44] or localizable entanglement [45]. This "best" ensemble is not always achievable, since one has to deal with physically realizable measurements $[10,28]$.

\section{References}

[1] M.A. Nielsen, I.L. Chuang, Quantum Computation and Quantum Information, Cambridge University Press, Cambridge, 2000.

[2] G. Benenti, G. Casati, G. Strini, Principles of Quantum Computation and Information, Vol. II, World Scientific, Singapore, 2007.

[3] M.B. Plenio, S. Virmani, Quantum Inf. Comput. 7 (2007) 1.

[4] R. Horodecki, P. Horodecki, M. Horodecki, K. Horodecki, Rev. Modern Phys. 81 (2009) 865.

[5] R. Lo Franco, B. Bellomo, E. Andersson, G. Compagno, Phys. Rev. A 85 (2012) 032318. 
[6] D. Rossini, G. Benenti, G. Casati, Phys. Rev. A 69 (2004) 052317;

B. Bellomo, R. Lo Franco, E. Andersson, J.D. Cresser, G. Compagno, Phys. Scr. T147 (2012) 014004;

R. Lo Franco, A. D’Arrigo, G. Falci, G. Compagno, E. Paladino, Phys. Scr. T147 (2012) 014019.

[7] C.H. Bennett, D.P. DiVincenzo, J.A. Smolin, W.K. Wootters, Phys. Rev. A 54 (1996) 3824.

[8] O. Cohen, Phys. Rev. Lett. 80 (1998) 2493.

[9] H. Nha, H.J. Carmichael, Phys. Rev. Lett. 93 (2004) 120408.

[10] A.R.R. Carvalho, M. Busse, O. Brodier, C. Viviescas, A. Buchleitner, Phys. Rev. Lett. 98 (2007) 190501.

[11] G. Gour, Phys. Rev. A 75 (2007) 054301.

[12] J. Eisert, T. Felbinger, P. Papadopoulos, M.B. Plenio, M. Wilkens, Phys. Rev. Lett. 84 (2000) 1611.

[13] M. Horodecki, P. Horodecki, R. Horodecki, Phys. Rev. Lett. 84 (2000) 2014.

[14] W.K. Wootters, Phys. Rev. Lett. 80 (1998) 2245.

[15] R. Alicki, K. Lendi, Quantum Dynamical Semigroups and Applications, in: Lect. Notes Phys., vol. 717, Springer, Berlin, Heidelberg, 2007.

[16] G. Falci, A. D’Arrigo, A. Mastellone, E. Paladino, Phys. Rev. Lett. 94 (2005) 167002.

[17] G. Ithier, E. Collin, P. Joyez, P.J. Meeson, D. Vion, D. Esteve, F. Chiarello, A. Shnirman, Y. Makhlin, J. Schriefl, G. Schön, Phys. Rev. B 72 (2005) 134519.

[18] J. Bylander, S. Gustavsson, F. Yan, F. Yoshihara, K. Harrabi, G. Fitch, D.G. Cory, Y. Nakamura, J.-S. Tsai, W.D. Oliver, Nat. Phys. 7 (2011) 565.

[19] F. Chiarello, E. Paladino, M.G. Castellano, C. Cosmelli, A. D’Arrigo, G. Torrioli, G. Falci, New J. Phys. 14 (2012) 023031.

[20] C.P. Slichter, Principles of Magnetic Resonance, Springer-Verlag, 1990.

[21] M.J. Biercuk, A.C. Doherty, H. Uys, J. Phys. B: At. Mol. Opt. Phys. 44 (2011) 154002.

[22] G.S. Uhrig, Phys. Rev. Lett. 98 (2007) 100504.

[23] H.-P. Breuer, E.-M. Laine, J. Piilo, Phys. Rev. Lett. 103 (2009) 210401.

[24] P. Meystre, M. Sargent III, Elements of Quantum Optics, fourth ed., Springer-Verlag, Berlin, 2007.

[25] R. Lo Franco, G. Compagno, A. Messina, A. Napoli, Phys. Rev. A 76 (2007) 011804(R).

[26] B. Bellomo, R. Lo Franco, G. Compagno, Phys. Rev. Lett. 99 (2007) 160502; Phys. Rev. A 77 (2008) 032342.

[27] C.E. López, G. Romero, F. Lastra, E. Solano, J.C. Retamal, Phys. Rev. Lett. 101 (2008) 080503;

C.E. López, G. Romero, J.C. Retamal, Phys. Rev. A 81 (2010) 062114;

Y.-K. Bai, M.-Y. Ye, Z.D. Wang, Phys. Rev. A 80 (2009) 044301.

[28] E. Mascarenhas, D. Cavalcanti, V. Vedral, M. França Santos, Phys. Rev. A 83 (2011) 022311.

[29] A. Rivas, S.F. Huelga, M.B. Plenio, Phys. Rev. Lett. 105 (2010) 050403.

[30] Y.S. Weinstein, T.F. Havel, J. Emerson, N. Boulant, M. Saraceno, S. Lloyd, D.G. Cory, J. Chem. Phys. 121 (2004) 6117.

[31] M. Gregoratti, R.F. Werner, J. Modern Opt. 50 (2003) 915.

[32] F. Buscemi, G. Chiribella, G.M. D’Ariano, Phys. Rev. Lett. 95 (2005) 090501.

[33] S. Vogelsberger, D. Spehner, Phys. Rev. A 82 (2010) 052327.

[34] A. Barchielli, M. Gregoratti, in: L. Accardi, F. Fagnola (Eds.), Quantum Probability and Related Topics, World Scientific, Singapore, 2012, arXiv:1202.2041 [quant-ph].

[35] K.W. Murch, S.J. Weber, C. Macklin, I. Siddiqi, Nature 502 (2013) 211.

[36] M. Mukhtar, T.B. Saw, W.T. Soh, J. Gong, Phys. Rev. A 81 (2010) 012331; M. Mukhtar, W.T. Soh, T.B. Saw, J. Gong, Phys. Rev. A 82 (2010) 052338.

[37] E. Paladino, Y.M. Galperin, G. Falci, B.L. Altshuler, Rev. Modern Phys. 86 (2014) 361.

[38] A. D’Arrigo, G. Benenti, R. Lo Franco, E. Paladino, G. Falci, Int. J. Quantum Inf. 12 (2014) 1461005.

[39] I. Devetak, IEEE Trans. Inform. Theory 51 (2005) 44.

[40] L. Mazzola, C.A. Rodriguez-Rosario, K. Modi, M. Paternostro, Phys. Rev. A 86 (2012) 010102(R).

[41] S. Luo, S. Fu, H. Song, Phys. Rev. A 86 (2012) 044101.

[42] H.J. Carmichael, An Open Systems Approach to Quantum Optics, Springer, Berlin, 1993.

[43] E. Mascarenhas, B. Marques, D. Cavalcanti, M. Terra Cunha, M. França Santos, Phys. Rev. A 81 (2010) 032310.

[44] D.P. DiVincenzo, C.A. Fuchs, H. Mabuchi, J.A. Smolin, A. Thapliyal, A. Uhlmann, Lecture Notes in Comput. Sci. 1509 (1999) 247.

[45] F. Verstraete, M. Popp, J.I. Cirac, Phys. Rev. Lett. 92 (2004) 027901; G. Gour, Phys. Rev. A 74 (2006) 052307. 